\title{
An evaluation of warfarin use at an urban district-level hospital in KwaZulu-Natal Province, South Africa
}

\author{
D J Laäs, MB ChB; M Naidoo, MB ChB, FCFP, MFamMed, MSc (Sports Medicine), PhD \\ Discipline of Family Medicine, School of Nursing and Public Health, College of Health Sciences, University of KwaZulu-Natal, Durban, South Africa
}

Corresponding author: D J Laäs (daniel.j.laas@gmail.com)

\begin{abstract}
Background. Warfarin is a commonly prescribed drug that needs to be monitored regularly.
Objectives. To evaluate the quality of care of patients on warfarin and to determine the cost-effectiveness of this drug in a South African district hospital setting.

Methods. Quality of care was assessed through a structured patient interview, and cost-effectiveness of warfarin was determined by retrospective review of all costs over a 6-month period.

Results. A total of 110 participants from the dedicated warfarin clinic at Wentworth Hospital, Durban, were enrolled over a 1-month period. The median cost of all warfarin-related expenditure was ZAR295.05 per patient per month (pppm) (interquartile range (IQR) 283.42 333.17), with a mean (standard error of the mean (SEM)) cost of ZAR394.90 (53.03). The median cost for patients with non-valvular atrial fibrillation was ZAR294.40 pppm (IQR 283.85 - 345.10) and the mean (SEM) cost was ZAR430.54 (116.14), compared with an estimated cost of ZAR545.96 and ZAR673.85 per month for rivaroxaban and dabigatran tablets, respectively. Of the participants interviewed ( $N=110)$, $69.1 \%$ agreed or strongly agreed that monthly international normalised ratio monitoring frustrated them, $74.5 \%$ agreed or strongly agreed that they sometimes wished that they did not have to take warfarin, and $84.5 \%$ would be willing to pay for a drug that required less frequent blood tests and clinic visits.

Conclusions. Warfarin still appears to be the most cost-effective drug in our setting, but it significantly affects patients' quality of life. We suggest a prospective head-to-head trial to compare both cost-effectiveness and quality of life of patients on warfarin and the new oral anticoagulants at a district hospital facility.
\end{abstract}

S Afr Med J 2018;108(12):1046-1050. DOI:10.7196/SAMJ.2018.v108i12.13256

Warfarin is an inexpensive and commonly used drug that is regularly prescribed in the public healthcare sector. Use of this drug is labour intensive, and patients in our setting undergo monthly international normalised ratio (INR) monitoring with regular dosing regimen adjustments. ${ }^{[1]}$ Difficulties associated with warfarin use include frequent drug interactions, drug-food interactions, and life-threatening complications due to subtherapeutic or excessively elevated INRs. ${ }^{[2,3]}$ Managing the patient on warfarin is complex, and with the added burden of complications increases the economic impact on the healthcare system and negatively affects patients' quality of life.

Large pharmacoeconomic studies have been conducted in the USA and Europe to evaluate the costs involved when prescribing warfarin. ${ }^{[4]}$ The relevance and applicability of these studies have become more apparent with the advent of the new or direct-acting oral anticoagulants (NOACs), which work by directly inhibiting thrombin or factor Xa. Warfarin inhibits the vitamin K-dependent synthesis of clotting factors II, VII, IX and X, as well as the regulatory factors protein $\mathrm{C}$ and protein $\mathrm{S} .{ }^{[5]}$ Warfarin use not only involves the direct costs of the drug and monthly INR monitoring, but additional costs in the form of supplementary hospital outpatient consultations, add-on bloods (full blood counts, renal and liver function tests), and sometimes the cost of inpatient care.

Wentworth Hospital (WWH), a public healthcare district hospital in Durban, South Africa (SA), has a dedicated outpatient warfarin clinic that is conducted every Friday. Unlike many other patients with chronic medical conditions who are seen 6-monthly when stable, patients on warfarin are seen at a maximum time interval of 1 month, and visits may occur weekly if the INR is found to be suboptimal.

\section{Objectives}

To evaluate the quality of care of patients on warfarin therapy presenting to an urban district hospital in KwaZulu-Natal Province, by assessing the financial and psychosocial burden of treatment.

\section{Methods}

This was a cross-sectional, observational, analytical study in which all patients who attended the warfarin clinic at WWH over a period of 4 weeks were interviewed to assess their quality of care. WWH is a busy urban district hospital in KwaZulu-Natal and the only district hospital in the south of eThekwini district. It has a catchment area with a population of $\sim 333740 .{ }^{[6]}$ According to WWH statistics for the 2016/17 financial year, 10600 outpatients were seen per month and 764 patients were admitted per month. An additional average of 989 patients visited the emergency department on a monthly basis. For the data collection period July - August 2017, there were six medical officers and two interns working in the outpatient department. The number of patients seen was 7334 for July and 7697 for August, with the average medical officer seeing 51.4 patients per day.

The participants' outpatient and inpatient files were retrospectively reviewed for the preceding 6-month period and all costs involved with each warfarin-related outpatient visit and inpatient stay were calculated. The maximum time for a follow-up appointment for these patients is 4 weeks, so the sample included all patients currently on warfarin therapy from the hospital. Patients attending the Friday clinic were included in the study, as well as patients admitted for complications arising from their warfarin use. Patients who were admitted after hours were also included in the study and their clinical 
files were retrieved from the admission ward. Patients presenting to the medical outpatient department for routine INR monitoring on any day but Friday as well as those with lost clinical files and those who had been on treatment for $<3$ months were excluded from the study. A patient's file was deemed to be lost if it could not be found on more than two separate occasions.

The study consisted of three components: a structured patient interview, a 6-month retrospective outpatient file review, and an inpatient file review of any admissions. The patient interview was further subdivided into two sections. Section A was used to verify whether a patient had been admitted to hospital during the past 6 months. The admission was reviewed to determine whether it was related to the patient's warfarin use, the date of admission and the use of blood or blood products. Questions in section B assessed the quality of care, patient expenses and patients' willingness to pay for alternatives. The questionnaire used in the interview was piloted at WWH and minor adjustments were made before use in the study. The piloted questionnaires were not included as part of the study population. The questionnaires were administered before each patient's consultation on their clinic day. It was administered by the first author, who asked the questions in English or Afrikaans. We employed a nursing assistant who assisted us with asking the questions in isiZulu. All patient responses were filled in by the data collectors.

The outpatient file review was aimed at determining the demographics and clinical profile of the patients, ascertaining outpatient costs, and finding objective evidence of warfarin-related hospital admissions. In determining the outpatient costs, the following information was sought: number of monthly warfarin tablets prescribed, number of blood investigations (including INRs) performed, and number of outpatient and emergency room visits.

Evidence of admission was based on one of the following criteria: an inpatient number, evidence of a discharge summary, evidence of admission in the outpatient notes, and review of the admission ward register. Once admission was confirmed, the inpatient notes were retrieved and key data elements were extracted. The inpatient review was aimed at determining healthcare costs such as the number of days admitted, use of blood and blood products, medications and costs of investigations.

Conservatism was the fundamental principle in determining costs. If there was doubt, either a specific cost was excluded or the less expensive option was chosen. Drug costs were obtained from the SV 35 Item Catalogue for the Provincial Pharmaceutical Supply Depot, date 1 June 2017. National Health Laboratory Service (NHLS) costs were obtained from the NHLS state price list for 2013. Blood and blood product costs were obtained from the South African National Blood Service state patient price list for 1 April 2016 - 31 March 2017. Inpatient costs were extrapolated from WWH statistics for the 2016/27 financial year, which estimate the cost of an inpatient day to be ZAR2 698. Outpatient consultation and emergency department costs were obtained from the 2017 hospital fees manual as there was no direct outpatient cost calculated by the hospital. These costs were calculated at the same value that a full-paying patient would pay, with the assumption made that this should cover $100 \%$ of all costs.

\section{Statistical analysis}

Data were extracted from the various source documents onto Microsoft Excel 2010 (Microsoft, USA) and imported in Stata version 13 (StataCorp, USA) for analysis. The costing data were found to be skewed and medians with interquartile ranges (IQRs) were used to interpret the data, with mean values (standard error of the mean (SEM)) used to determine individual and total costs. Statistical analysis was conducted with the assistance of a statistician from the
College of Health Sciences, University of KwaZulu-Natal. The study population was divided into male and female and above and below 60 years of age. The age 60 was chosen to separate the population into young and old, with a patient aged $\geq 60$ years being classified as elderly. A $p$-value $<0.05$ was considered significant.

Quality of care data were converted from categorical to numerical data. Means and medians were used to interpret data with standard deviations (SDs) and IQRs used to determine data spread.

\section{Ethical considerations}

Permission to conduct this study was obtained from the Biomedical Research Ethics Committee, University of KwaZulu-Natal (ref. no. BE364/17), the KwaZulu-Natal Department of Health's ethical review board (ref. no. KZ_2017RP4_664) and the CEO of WWH. Written informed consent was individually obtained from every patient included in the study.

\section{Results}

A total of 128 patients were eligible for the study, of whom 18 (14.1\%) were excluded, giving a final number of 110 participants. A total of 135 patients were booked for the clinics for the study period. Of the patients excluded, 9 had been on treatment for $<3$ months and 9 patients' files were not found.

Table 1 illustrates the relationship between age and a number of variables measured.

There was a statistically significant difference between respondents under and over 60 years of age with regard to atrial fibrillation (AF), prosthetic heart valves and hypertension. The prevalences of AF and hypertension were higher in patients aged $>60$ years, while prosthetic heart valves were more common in patients aged $<60$ years.

The total cost of all expenses related to warfarin per patient per month (pppm) over a 6-month period was calculated as ZAR394.98 and is tabulated with other costs in Table 2.

The total cost attributed to warfarin for all patients over a 6-month period was ZAR260 628.83, with the different cost components illustrated in Fig. 1.

Warfarin $5 \mathrm{mg}$ tablet costs, INR costs, costs of additional blood investigations and outpatient visits amounted to a total outpatient cost of ZAR204 014.33. Admissions resulted in a total inpatient cost of ZAR56 614.50. The total number of outpatient INRs performed amounted to 741, with an average of 6.7 INRs per patient over 6 months. Urea, creatinine and electrolytes was the most prevalent additional investigation done $(n=34)$, followed by full blood counts $(n=28)$, liver function tests $(n=19)$, glycated haemoglobin $(n=12)$, lipograms $(n=10)$, thyroid function tests $(n=6)$, prostate-specific antigen tests $(n=5)$ and calcium, magnesium and phosphate $(n=4)$.

A total of 717 chronic outpatient, 6 accident and emergency and 10 acute outpatient visits were directly related to warfarin use. These visits consisted of routine INR monitoring or complications of an elevated or subtherapeutic INR. Three patients who fulfilled our inclusion criteria were admitted to hospital. Three inpatients were excluded because they had been on treatment for $<3$ months or their files were missing. One patient was admitted for a subtherapeutic INR and 2 were admitted for warfarin toxicity with an elevated INR. One patient was treated for warfarin toxicity in the emergency department and discharged within 24 hours. The mean inpatient stay for the $2.7 \%$ of patients who required admission for a warfarinrelated complication over the study period was 6.3 days. Three patients required admission for non-warfarin-related indications, and the costs of these admissions were not included in the costs of warfarin use. Table 3 lists patients' satisfaction with the quality of care received. 


\begin{tabular}{|c|c|c|c|c|}
\hline & \multicolumn{2}{|c|}{ Age (years) } & \multirow[b]{2}{*}{ Total $(N)$} & \multirow[b]{2}{*}{$p$-value } \\
\hline & $<60(N=53), n(\%)$ & $>60(N=57), n(\%)$ & & \\
\hline \multicolumn{5}{|l|}{ Sex } \\
\hline Male & $24(45.3)$ & $26(45.6)$ & 50 & 0.9 \\
\hline Female & $29(54.7)$ & $31(54.4)$ & 60 & \\
\hline \multicolumn{5}{|l|}{ Indication } \\
\hline $\mathrm{AF}$ & $9(17.0)$ & $33(57.9)$ & 42 & $<0.001$ \\
\hline Prosthetic heart valve & $33(62.3)$ & $14(24.6)$ & 47 & $<0.001$ \\
\hline DVT & $5(9.4)$ & $4(7.0)$ & 9 & 0.6 \\
\hline PE & $3(5.7)$ & $1(1.8)$ & 4 & 0.4 \\
\hline Other & $4(7.5)$ & $5(8.8)$ & 9 & 0.8 \\
\hline \multicolumn{5}{|l|}{ Comorbidities } \\
\hline Diabetes & $6(11.3)$ & $17(29.8)$ & 23 & 0.02 \\
\hline Prosthetic heart valve with comorbid AF & $9(17.0)$ & $7(12.3)$ & 16 & 0.5 \\
\hline Hypertension & $16(30.2)$ & $40(70.2)$ & 56 & $<0.001$ \\
\hline Dyslipidaemia & $4(7.5)$ & $14(24.6)$ & 18 & 0.02 \\
\hline IHD & $1(1.9)$ & $10(17.5)$ & 11 & 0.009 \\
\hline Cardiac failure & $3(5.7)$ & $15(26.3)$ & 18 & 0.003 \\
\hline COPD & $1(1.9)$ & $3(5.3)$ & 4 & 0.6 \\
\hline Asthma & $3(5.7)$ & $3(5.3)$ & 6 & 0.9 \\
\hline Other & $24(45.3)$ & $19(33.3)$ & 43 & 0.2 \\
\hline \multicolumn{5}{|l|}{ Comorbidities, $N$} \\
\hline 0 & $17(32.1)$ & $6(10.5)$ & 23 & 0.005 \\
\hline 1 & $14(26.4)$ & $12(21.1)$ & 26 & 0.508 \\
\hline 2 & $17(32.1)$ & $15(26.3)$ & 32 & 0.506 \\
\hline$\geq 3$ & $5(9.4)$ & $24(42.1)$ & 29 & $<0.001$ \\
\hline \multicolumn{5}{|l|}{ Time spent at hospital (hours) } \\
\hline $0-<5$ & $16(30.2)$ & $13(22.8)$ & 29 & 0.38 \\
\hline$\geq 5-<6$ hours & $14(26.4)$ & $32(56.1)$ & 46 & 0.002 \\
\hline$\geq 6$ & $23(43.4)$ & $12(21.1)$ & 35 & 0.012 \\
\hline \multicolumn{5}{|l|}{ Willingness to pay } \\
\hline Not willing to pay & $9(17.0)$ & $8(14.0)$ & 17 & 0.669 \\
\hline ZAR0 - 49 & $16(30.2)$ & $28(49.1)$ & 44 & 0.04 \\
\hline ZAR50 - 99 & $17(32.1)$ & $15(26.3)$ & 32 & 0.506 \\
\hline ZAR100 - 199 & $8(15.1)$ & $3(5.3)$ & 11 & 0.086 \\
\hline$\geq$ ZAR200 & $3(5.7)$ & $3(5.3)$ & 6 & 0.927 \\
\hline
\end{tabular}

\section{Table 2. Costs of prescribing warfarin}

\begin{tabular}{llll}
\hline & Patients $(N)$ & ZAR & IQR/SEM \\
\hline Total costs pppm & & & \\
$\quad$ Median (IQR) & 110 & 295.05 & $283.42-333.17$ \\
$\quad$ Mean (SEM) & 110 & 394.89 & 53.03 \\
Non-valvular AF costs pppm & 42 & 294.40 & $283.85-345.10$ \\
$\quad$ Median (IQR) & 42 & 430.54 & 116.14 \\
$\quad$ Mean (SEM) & & & \\
Other indications pppm & 68 & 296.13 & $279.97-330.2$ \\
$\quad$ Median (IQR) & 68 & 372.87 & 47.69 \\
$\quad$ Mean (SEM) & 4 & & \\
Inpatient and emergency department cost pp 6 mo & 4 & 12141.00 & $5385.13-22922.13$ \\
$\quad$ Median (IQR) & & 14153.63 & 5866.00 \\
$\quad$ Mean (SEM) & 110 & 1764.80 & $1690.83-1982.22$ \\
Outpatient cost pp 6 mo & 110 & 1854.68 & 46.37 \\
$\quad$ Median (IQR) & &
\end{tabular}




\begin{tabular}{|c|c|c|c|}
\hline & Mean (SD) & Median & IQR \\
\hline Treatment satisfaction & $3.89(1.03)$ & 4 & $4-5$ \\
\hline I am often frustrated with having to come to the hospital every month for blood tests & $3.75(1.22)$ & 4 & $2-5$ \\
\hline I sometimes wish that I did not have to take warfarin & $4(1.18)$ & 4 & $3-5$ \\
\hline Willing to pay for an alternative drug & $3.82(0.84)$ & 4 & $2-4$ \\
\hline
\end{tabular}

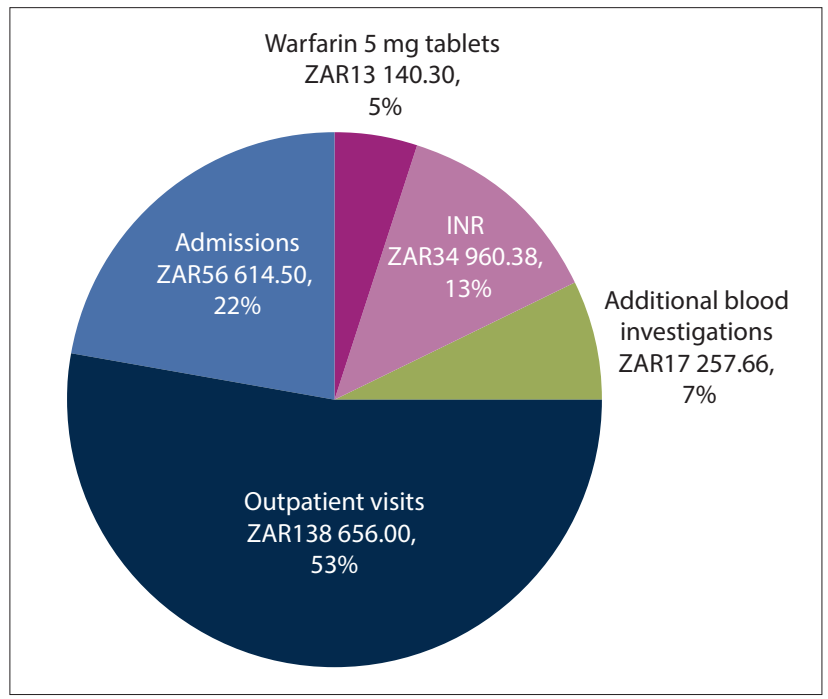

Fig. 1. Total cost of warfarin. (INR = international normalised ratio.)

The quality of care analysis also revealed that $80.0 \%$ of participants agreed or strongly agreed that they were satisfied with the care they received, $69.1 \%$ agreed or strongly agreed that monthly INR monitoring frustrated them, and $74.5 \%$ agreed or strongly agreed that they wished they did not have to take warfarin.

The monthly mean (SEM) transportation cost per patient was calculated at ZAR57.20 (6.89). Of the patients, $84.5 \%$ would be willing to pay for a drug that required less monitoring and fewer clinic visits; however, $47.3 \%$ of patients willing to pay could only afford ZAR0 - 50 per month.

\section{Discussion}

\section{Main findings}

From the main study findings, it was concluded that warfarin affects the quality of life of our patient population to the extent that many patients would be willing to pay for an alternative drug. In addition, we found that warfarin is a cost-effective treatment option in our setting. Outpatient consultations, admission costs and expenses incurred by patients with non-valvular AF accounted for the largest proportion of expenses.

\section{Other findings}

The majority of patients were satisfied with the overall quality of care they received at WWH. Many commented that having a specific clinic day and a dedicated warfarin clinic greatly contributed to this. Unfortunately, this does not offset the effect that monthly consultations and blood investigations has on the patient population, with over two-thirds of the patients reporting that they wished they did not have to take this drug. In addition, the majority of patients spent 5 - 6 hours at hospital awaiting blood results and medication, and spent ZAR57.20 on getting to and from the hospital every month. In a population that consists mostly of the elderly, pensioners and the unemployed, this transportation cost is considered to be excessive.
Individuals who receive a government pension of ZAR1 600 per month spend on average ZAR686.4 per year on transportation. ${ }^{[7]}$

In a health-related quality of life analysis from the RE-LY trial, Monz et al ${ }^{[8]}$ compared the quality of life of patients on dabigatran with those on warfarin. The visual analogue scale of the EQ-5D questionnaire (range 0 - 100, with 100 being best imaginable health) among others was used to determine this and found that after 12 months of treatment with warfarin the mean score was 74.0 (range 71.6 - 76.5). ${ }^{[9]}$ These results contrast with our results, and the reasons for this difference are thought to be multifactorial. We believe that the underlying frustrations of our patients can be attributed to the pain of monthly venepunctures, long waiting times and additional financial burdens. With these frustrations in mind, we asked our patients if they would be willing to pay for a drug that requires fewer clinic visits and less monitoring, to which a substantial majority agreed. This is significant in view of our study population's already poor socioeconomic circumstances. These circumstances are highlighted by the largest proportion of patients stating that they would only be able to afford ZAR0 - 50 per month for an alternative drug.

Our data collection showed that AF is a disease of the elderly and that prosthetic heart valves are more prevalent in individuals aged $<60$ years. The decision to divide the population into over and under 60 years was based on data from the Framingham Study, which suggested that the risk of stroke from AF increased significantly across the decades after 50 - 59 years of age ${ }^{[10]}$ We found a statistically significant prevalence of hypertension in participants aged $>60$ years, which can be linked to the increased incidence of AF in the same age group. This result is comparable to other studies that emphasise the need for early cardiovascular risk factor control and prevention, as an increase in age and hypertensive heart disease are major risk factors for the development of $\mathrm{AF}^{[11]}$ The demographics and risk factor profile in our study are similar to an SA study by Sonuga et al. ${ }^{[12]}$ They found hypertension to be the commonest comorbidity, and the patient population consisted of more females than males. A notable difference is that the commonest indication for warfarin was $\mathrm{AF}$ and not prosthetic heart valves as in our study. A reason for this may be that many patients had valve replacements at WWH, as cardiothoracic surgery used to be based there. ${ }^{[12]}$

We designed our costing model to include all costs involved when prescribing warfarin, such as additional blood investigations performed at the monthly clinic visit. From a costing perspective, we found two broad groups as illustrated by the non-linear spread of data: those requiring admission and those not requiring admission. We found no significant difference between the total median cost pppm, the median cost of non-valvular AF (NVAF) pppm and other indications for warfarin pppm. AF appears to be a main driver of costs, with significant differences between the average cost of NVAF pppm in comparison with the average cost of other indications. These differences in the average costs between AF and non-AF patients can be attributed to the AF patients' admission costs and additional blood investigations. This finding is in keeping with an economic analysis conducted in the UK, where admission fees contributed to $50 \%$ of total AF costs. ${ }^{[13]}$ 
After determining the costs of warfarin, we contacted the manufacturers of dabigatran, Boehringer-Ingelheim South Africa, and rivaroxaban, Bayer South Africa, in an attempt to determine what the cost price of these drugs would be in the state sector. Depending on factors such as volume sold, Boehringer-Ingelheim estimated that dabigatran would cost the National Department of Health between ZAR673.85 and ZAR700.80 for a month's supply. Bayer estimated that the cost of rivaroxaban would be between $50 \%$ and $75 \%$ of the current single exit price. The medicine price registry lists the maximum single exit price of rivaroxaban $20 \mathrm{mg}$ at ZAR1 091.92 (including VAT and dispensing fees) for a month's supply. ${ }^{[14]}$ Using this figure, the cost of rivaroxaban tablets alone would be between ZAR545.96 and ZAR818.94 per patient per month. These costs exclude event costs such as admissions, blood products and additional blood investigations.

The ROCKET-AF, ARISTOTLE and RE-LY studies found the NOACs to be non-inferior to and in some cases superior to warfarin. ${ }^{[15-}$ ${ }^{17]}$ Dabigatran $150 \mathrm{mg}$ twice daily has a stroke or systemic embolism rate of $1.11 \%$ per year for patients with NVAF compared with $1.69 \%$ per year for patients on dose-adjusted warfarin $(p<0.001)$. The rates of major bleeding for dabigatran $150 \mathrm{mg}$ and dose-adjusted warfarin appear to be similar, but there is a significantly decreased risk of life-threatening intracranial bleeds with dabigatran $110 \mathrm{mg}(0.12 \%$; $p<0.001)$ and $150 \mathrm{mg}(0.10 \% ; p<0.001)$ in comparison with warfarin $(0.38 \%) .{ }^{[17]}$ Rivaroxaban showed similar results, with a significant reduction in fatal bleeds in comparison with warfarin $(0.2 \% \mathrm{v} .0 .5 \%$; $p=0.003) .{ }^{[15]}$ Rivaroxaban also appears to be more effective than aspirin in the prevention of secondary cardiovascular events. ${ }^{[18]}$

As a result of these findings, pharmacoeconomic studies were conducted using Markov decision models in an attempt to evaluate the cost-effectiveness of the NOACs. These studies found that the NOACs are a cost-effective alternative when compared with doseadjusted warfarin. ${ }^{[4,19,20]}$ Bergh et al. ${ }^{[2]}$ conducted a similar costeffectiveness analysis in the SA private sector and came to the same conclusion as the international studies.

We believe that our results differ from the international literature because of our setting and the availability of generic medication. Patients at WWH are seen by medical officers at district hospital level, which in combination with the relatively low cost of warfarin $5 \mathrm{mg}$ tablets significantly decreases costs.

\section{Study limitations}

Assessment of cost-effectiveness was challenging owing to difficulties in determining outpatient consultation costs, missing files and a paper filing system. Some admitted patients were excluded because files were missing, and if these costs were included they could add a substantial amount to the total costs per patient per month. As this was a cross-sectional study, we were only able to give a 6-month snapshot of the various social and economic factors influencing patients on warfarin, which makes it difficult to draw accurate conclusions. We believe that a longer study time frame would influence both cost-effectiveness and quality of care data. Unfortunately, a retrospective review of more than 6 months will be difficult owing to the challenges associated with a paper filing system, missing files and recall bias.

Other study limitations include using only one site for data collection and using subjective measures to gauge socioeconomic status and income.

\section{Conclusions}

We found that warfarin has an adverse effect on our patients' quality of life, but it is still the most cost-effective anticoagulant in our setting. The NOACs will only be a cost-effective alternative in a district health setting if the current estimated public sector drug prices for both rivaroxaban and dabigatran are approximately halved. One cannot ignore the pharmacological benefits and convenience of the NOACs, together with the psychosocial disadvantages of warfarin. On this basis we suggest a prospective head-to-head trial that compares both cost-effectiveness and quality of life of patients on warfarin and NOACs at a district health level.

Introduction of these new agents has the potential to improve quality of care for all patients on anticoagulation treatment. For those still on warfarin it will decrease waiting times and allow for more doctor/patient contact, while those on a NOAC will need fewer clinic visits and blood investigations. This will ultimately decrease the congestion at district hospitals.

Declaration. This publication was a requirement for the DJL's Master of Medical Science degree.

Acknowledgements. The authors acknowledge the contributions of Dr R Laäs and Dr B Oyebola, and the staff of WWH for their support and assistance with the study.

Author contributions. DJL was the principal investigator and MN was the research supervisor.

Funding. A research bursary was obtained from the South African Medical Association. The authors state that the bursary had no influence on the outcome of the study.

Conflicts of interest. None.

1. Rose AJ, Ozonoff A, Berlowitz DR, Ash AS, Reisman JI, Hylek EM. Reexamining the recommended follow-up interval after obtaining an in-range international normalized ratio value: Results from the Veterans Affairs Study to Improve Anticoagulation. Chest 2011;140(2):359-365. https://do org $/ 10.1378 /$ chest.10-2738

2. Shameem R, Ansell J. Disadvantages of VKA and requirements for novel anticoagulants. Best Pract Res Clin Haematol 2013;26(2):103-114. https://doi.org/10.1016/j.beha.2013.07.009

3. Kapp PA, Klop AC, Jenkins LS. Drug interactions in primary health care in the George subdistrict, South Africa: A cross-sectional study. S Afr Fam Pract 2013;55(1):78-84. https://doi.org/10.1080/20 South Africa: A cross-
786204.2013.10874307

4. Coyle D, Coyle K, Cameron C, et al. Cost-effectiveness of new oral anticoagulants compared with Coyle D, Coyle K, Cameron C, et al. Cost-effectiveness of new oral anticoagulants compared with
warfarin in preventing stroke and other cardiovascular events in patients with atrial fibrillation. Value warfarin in preventing stroke and other cardiovascular events in patients
Health 2013;16(4):498-506. https://doi.org/10.1016/j.jval.2013.01.009

5. Ansell J, Hirsh J, Hylek E, Jacobson A, Crowther M, Palareti G. Pharmacology and management of 5. Ansell J, Hirsh J, Hylek E, Jacobson A, Crowther M, Palareti G. Pharmacology and management of
the vitamin K antagonists. Chest 2008;133(6 Suppl):160-198. https://doi.org/10.1378/chest.08-0670

the vitamin K antagonists. Chest 2008;133(6 Suppl):160-198. https://doi.org/10.1378/chest.08-0670
6. Department of Health, Province of KwaZulu-Natal. Wentworth Hospital. http://www.kznhealth.gov. za/wentworthhospital.htm (accessed 29 October 2018).

7. South African Government. https://www.gov.za/services/social-benefits-retirement-and-old-age/oldage-pension (accessed 29 October 2018).

8. Monz BU, Connolly SJ, Korhonen M, Noack H, Pooley J. Assessing the impact of dabigatran and warfarin on health-related quality of life: Results from an RE-LY sub-study. Int J Cardio 2013;168(3):2540-2547. https://doi.org/10.1016/j.ijcard.2013.03.059

9. Van Reenen M, Janssen B. EQ-5D-5L user guide. Basic Inf how to use EQ-5D-5L. Version 2.1. Rotterdam: EuroQol Research Foundation, April 2015. https://euroqol.org/wp-content/ uploads/2016/09/EQ-5D-5L_UserGuide_2015.pdf (accessed 5 November 2018).

10. Wolf PA, Abbott RD, Kannel WB. Atrial fibrillation as an independent risk factor for stroke: The Framingham Study. Stroke 1991;22(8):983-988. https://doi.org/10.1161/01.STR.22.8.983 11. Kannel WB, Abbott RD, Savage DD, McNamara PM. Epidemiologic features of chronic atrial
fibrillation: The Framingham study. N Engl J Med 1982;306(17):1018-1022. https://doi.org/10.1056/ NEJM198204293061703

12. Sonuga BO, Hellenberg DA, Cupido CS, Jaeger C. Profile and anticoagulation outcomes of patients on warfarin therapy in an urban hospital in Cape Town, South Africa. Afr J Prim Health Care Fam Med 2016;8(1):el-e8. https://doi.org/10.4102/phcfm.v8i1.1032

13. Stewart S. Cost of an emerging epidemic: An economic analysis of atrial fibrillation in the UK. Heart 2004;90(3):286-292. https://doi.org/10.1136/hrt.2002.008748

14. Code for South Africa. What should your medicines cost? https://mpr.code4sa.org/\#search:rivaroxaban (accessed 29 October 2018).

5. Patel MR, Mahaffey KW, Garg J, et al. Rivaroxaban versus warfarin in nonvalvular atrial fibrillation (ROCKET AF trial). N Engl J Med 2011;365:883-891. https://doi.org/10.1056/NEJMoa1009638

16. Granger $\mathrm{CB}$, Alexander JH, McMurray JJV, et al. Apixaban versus warfarin in patients with atrial fibrillation. N Engl J Med 2011;365(11):339-354. https://doi.org/10.1056/NEJMoa1107039

17. Connolly SJ, Ezekowitz MD, Yusuf S, et al. Dabigatran versus warfarin in patients with atrial fibrillation. N Engl J Med 2009;361(12):1139-1151. https://doi.org/10.1056/NEJMoa0905561

8. Eikelboom JW, Connolly SJ, Bosch J, et al. Rivaroxaban with or without aspirin in stable cardiovascula disease. N Engl J Med 2017;377:1319-1330. https://doi.org/10.1056/NEJMoa1709118

19. Harrington AR, Armstrong EP, Nolan PE, Malone DC. Cost-effectiveness of apixaban, dabigatran, rivaroxaban, and warfarin for stroke prevention in atrial fibrillation. Stroke 2013;44(6):1676-1681. https://doi.org/10.1161/STROKEAHA.111.000402

20. Lee S, Anglade MW, Pham D, Pisacane R, Kluger J, Coleman CI. Cost-effectiveness of rivaroxaban compared to warfarin for stroke prevention in atrial fibrillation. Am J Cardiol 2012;110(6):845-851. compared to warfarin for stroke prevention in
https://doi.org/10.1016/j.amjcard.2012.05.011

21. Bergh M, Marais CA, Miller-Janson H, Salie F, Stander MP. Economic appraisal of dabigatran as firstline therapy for stroke prevention in atrial fibrillation. S Afr Med J 2013;103(4):241-245. https://doi. org/10.7196/SAMJ.6471

Accepted 4 June 2018. 